\title{
Decision and Risk Analysis for an Air Carrier under Fuzzy Conditions
}

\author{
Souhir CHARFEDDINE \\ ENAC (French Civil Aviation Institute) \\ LARA(Laboratory of Automatics and Applied operational Research), \\ charfeddinesouhir@yahoo.fr \\ Felix MORA-CAMINO \\ ENAC \\ Felix.mora-camino@enac.fr \\ Marc De Coligny \\ UT2 (University of Toulouse II) \\ Coligny@univ-tlse2.fr \\ Karim ZBIDI \\ ENAC and UT2 \\ zbidi@recherche.enac.fr
}

\begin{abstract}
In the Air Transport sector where the uncertainty is very frequent due to the high sensitivity of the activity to many factors that influence the behavior of the consumers. Fuzzy logic can be used to produce estimates which take into account the vagueness of the operating environment. In this sector, many important decisions about the supply of service are based on these estimates.

In this paper an analysis of the optimal supply conditions under fuzzy demand information is considered for a market where only one carrier is operating. The carrier's supply decision making process is investigated, first when the demand function is obtained through classical methods then when it is given by a fuzzy function. The main advantages of the new approach are then discussed.
\end{abstract}

\section{Introduction}

Air transport markets are characterized by very tight margins of profit. Besides that, the demand for these markets is very sensitive to many variables (wars, economic crisis, cews strikes,...). So an airline needs an efficient decision making system in order to make positive profits. The decision making and the choice of the strategies for an operator such as the maximization of his profit need estimation of his own demand and of the demand of the possible competitors. This estimation of the demand is in general and in the classical approach, obtained through econometric regressions based on historical data and through statistical methods, leading to the definition of a crisp demand function. Some fuzzy modeling techniques ([9] and [3]) have recently been developed to take into account the uncertainty related with these predictions, leading to the definition of fuzzy demand functions. This last approach allows in particular introducing more uncertainty in the estimation of demand of the competitor as it happens in general due to the lack of direct information.

Through the decision process, the fuzziness of demand propagates to the profit estimation. A fuzzy optimization process is then necessary to generate sets of possible solutions.

Here an air transport market is considered where it is supposed that there is only one carrier operating so that we focus on the fuzzy nature of the estimation of the demand. The decision here consists to choose the fare and the frequency of flights to put on the market for a given period of time. 
Starting from the analysis of the decision making process in the deterministic case, a new decision making process is designed to cope with a fuzzy estimation of the demand function.

\section{Deterministic demand function model}

Let $F$ denote the set of possible frequency levels ( $F$ is a finite set of positive integers). The demand function is supposed to be increasing with respect to frequency and decreasing with respect to fare. A possible model is given by:

$$
\begin{array}{r}
\text { for } p_{\min } \leq p \leq p_{\max } \text { and } f \in F, \\
D(p, f)=D_{0}-\lambda(p-\varphi(f))
\end{array}
$$

where $\quad \mathrm{D}_{0}$ and $\lambda$ are strictly positive parameters, $\mathrm{D}_{0}$ is the potential gross demand, while $\lambda *(p-\varphi(f))$ (supposed to be a positive entity) is the degradation of the demand level resulting from the offer of the airline (generalized cost composed by the price and cost of the time of travel expressed as a function of the frequency).

$\varphi$ is an increasing and strictly concave function, for example $\varphi(f)=\frac{-\tau}{f}$ with $\tau>0$

$\left[p_{\min }, p_{\max }\right]$ is the interval of possible fare values.

The operational cost on this market is supposed to be linear with respect to the frequency and to the level of the satisfied demand. A classical model [8] which is used in this study is given by:

$$
C(p, f)=c_{0}+c_{f} \cdot f+c_{d} \min \{D(p, f), K . f\}
$$

where

- $\mathrm{c}_{0}$ is the fixed operations cost for the considered market

- $\quad \mathrm{c}_{f}$ traduces the increase of cost resulting from the establishment of a new frequency,

- $\quad \mathrm{c}_{\mathrm{d}}$ is the marginal cost related to an additional transported passenger and

- $\mathrm{K}$ is the capacity of the fleet of airplanes disserving the city pair O-D, this fleet is supposed to be homogenous.

The profit resulting from the operation on this single origin-destination market can then be written as:

$$
\pi=\left(p-c_{d}\right) \min \{D(p, f), K . f\}-c_{0}-c_{f} \cdot f
$$

The target of the airline is to choose a fare $p$ from a possible set $\left[p_{\min }, p_{\max }\right] \subset \boldsymbol{R}$ and the frequency $f$ $(\in F)$ that maximize this profit. Since it is easier to change fares than to change frequencies, a current practice is to solve this problem in two stages: first the frequency is taken as a parameter while the fare is chosen as a function of it and then the frequency is optimized as in [1]. For a given, level of $f$, the problem of the carrier becomes:

$$
\underset{p}{\operatorname{Max}} \pi=\left(p-c_{d}\right) \min \{D(p, f), K . f\}-c_{0}-c_{f} . f .
$$

The search for a maximum profit leads at first sight to consider the cases where $D(p, f)<K$.f and where $D(p, f) \geq K . f$. When $D(p, f) \geq K . f$, the level of the satisfied demand is exactly equal to $K f$, then the profit of the airline is given by the expression: $\pi=\left(p-c_{d}\right) . K \cdot f-c_{0}-c_{f} \cdot f$.

Since according to this expression the profit is increasing with respect to the fare $p$, the fare will be chosen as high as possible, this will be achieved when the fare is such that: ' $D(p, f)=K . f$ '. So the two initial cases can be grouped under the unique condition: $D(p, f) \leq K . f$. Then the expression (1) of the profit can be reduced to:

$$
\pi=\left(p-c_{d}\right) \cdot D(p, f)-c_{0}-c_{f} \cdot f
$$

With this last expression, it is possible to use the classical optimality conditions to determine the optimum solution; the first order condition of Lagrange is then given by:

$$
\partial \pi / \partial p=D_{0}+\lambda c_{d}-2 \lambda p+\lambda \varphi(f)
$$

while the second order condition, in fact the concavity condition for $\pi$ can be traduced by:

$$
\partial^{2} \pi / \partial p^{2}<0
$$

Observe that this last condition is always satisfied since here: $\partial^{2} \pi / \partial p^{2}=-2 \lambda$.

So for every feasible $f \in F$, there is an optimal fare $p_{f}^{*}$ that maximizes the profit $\pi$. The resulting optimized profit can be denoted as: $\pi_{f}^{*}=\pi\left(p_{f}^{*}, f\right)$ where $p_{f}^{*}$ is given by:

$$
p_{f}^{*}=c_{d} / 2+D_{0} /(2 \lambda)+\varphi(f) / 2
$$


For this two step approach, the retained frequency $f^{*}$ that will be such as:

$$
f^{*}=\underset{f \in F}{\arg \max }\left\{\pi_{f}^{*}, f \in F\right\}
$$

where

$$
\pi_{f}^{*}=\left(D_{0}+\lambda \varphi(f)+\lambda c_{d}\right)^{2} / 4 \lambda-c_{f} f-c_{0}
$$

Then the optimal price will be given by:

$$
p^{*}=c_{d} / 2+D_{0} /(2 \lambda)+\varphi\left(f^{*}\right) / 2
$$

Observe that the solution obtained through these two stages is a global solution for the problem. This is due to the fact that $\mathrm{F}$ is a finite set and all the cases are then explored.

In practical grounds it appears that this approach is difficult to be implemented since the adopted demand model provides estimates that are "around" the observed values, but with uncontrolled variations. This is mainly a consequence of the dependency of the demand levels to many qualitative factors and an intensification of statistical efforts may result no appreciable improvement of the accuracy of the estimates.

\section{The case of a Fuzzy demand function Main title}

In this subsection, it is then assumed that the available estimates of demand for the airline are represented by fuzzy sets allowing the inclusion of qualitative considerations in the building of these estimates: a fuzzy demand function is obtained. For every configuration (price, frequency), a fuzzy set is associated (and not only one crisp value as in the classical approach). The basis of the fuzzy set represents the possible values of the demand relative to this configuration. They can be obtained from the subjective beliefs of the experts which are mainly based on past experience and data [Charfeddine et al., 2004]. The membership function of this fuzzy set can take different shapes: triangular, trapezoidal. This fuzzy set has to cover the main possibilities which can result from different attitudes (optimistic, medium and pessimistic ones).

\subsection{A fuzzy demand function model}

For a given couple, $(p, f) \in\left[p_{\min }, p_{\max }\right] \times F$ the associated fuzzy estimate of demand $\tilde{D}(p, f)$ is here assumed to be represented by a trapezoidal fuzzy number and the fuzzy function $\tilde{D}$ is defined by four level 'crisp' functions $D^{a}, D^{b}, D^{c}$ and $D^{d}$.

Then, choosing a frequency $f$ in $F$, a fuzzy function of price, parameterized by the frequency can be defined by: $\widetilde{D}_{f}(p)=\tilde{D}(p, f)$ and is then composed of four "level functions" in the sense of Zimmermann [10]. Figure 2 sketches such a fuzzy function by showing for the interval $\left[p_{\min }, p_{\max }\right]$ "level functions" of $\tilde{D}_{f}(p)$. There $D_{f}^{b}$ and $D_{f}^{c}$ are the functions associated to a degree of membership equal to $1 . D_{f}^{a}$ and $D_{f}^{d}$ are the functions associated to the limits of the base of the corresponding fuzzy numbers ( the membership degree starts there from zero). Consistence implies that these four functions cannot intersect each other over the $\operatorname{domain}\left[p_{\min }, p_{\max }\right]$.

For every price $p$ in the allowed domain, the membership function $\mu_{D_{f}}^{p}$ of the demand is, according to the trapezoidal assumption, so that $\mu_{D_{f}}^{p}$ (d) is equal to:

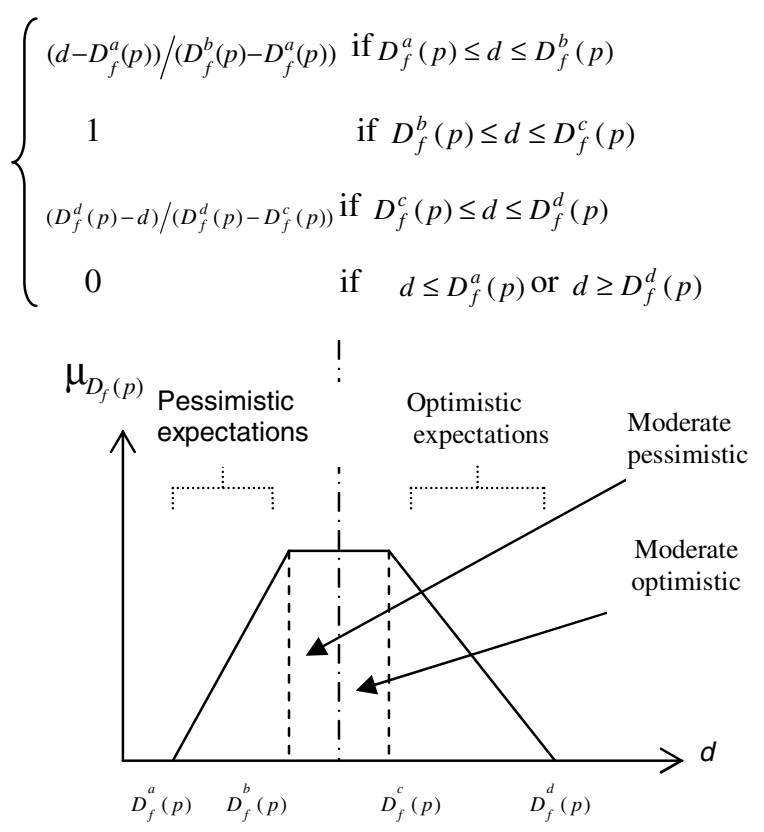

Figure 1. Trapezoidal membership function 
Here the function $D^{b}$ can be associated to pessimistic expectations and $D^{c}$ to optimistic ones, it is much expected that the effective value of demand will be within their range, while $D^{a}$ is associated to extremely pessimistic expectations and $D^{d}$ to extremely optimistic ones.

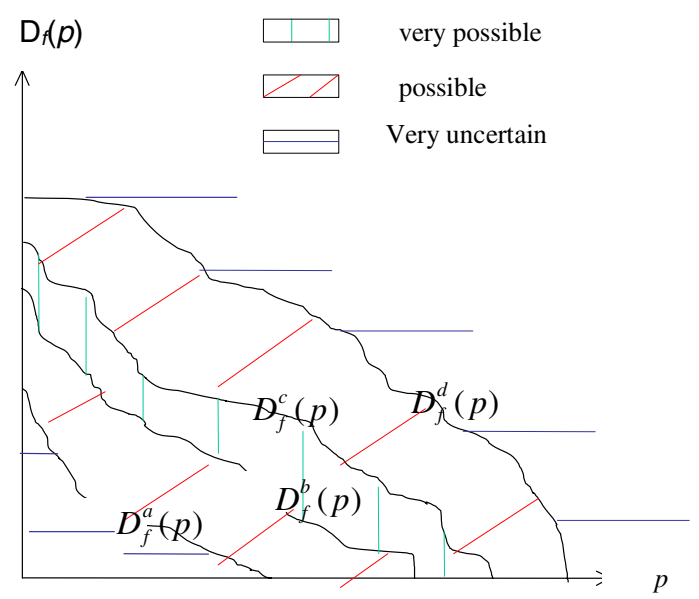

Figure2. Fuzzy demand function

It is further assumed that the level functions adopts model (1) with different parameters values:

For every $k \in\{a, b, c, d\}$,

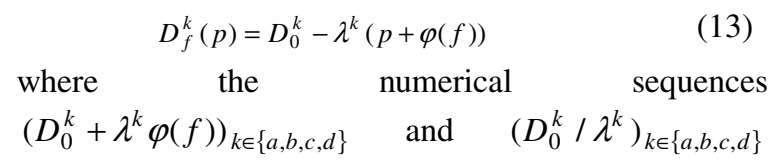
increase with $\mathrm{k}$ varying along $\{a, b, c, d\}$.

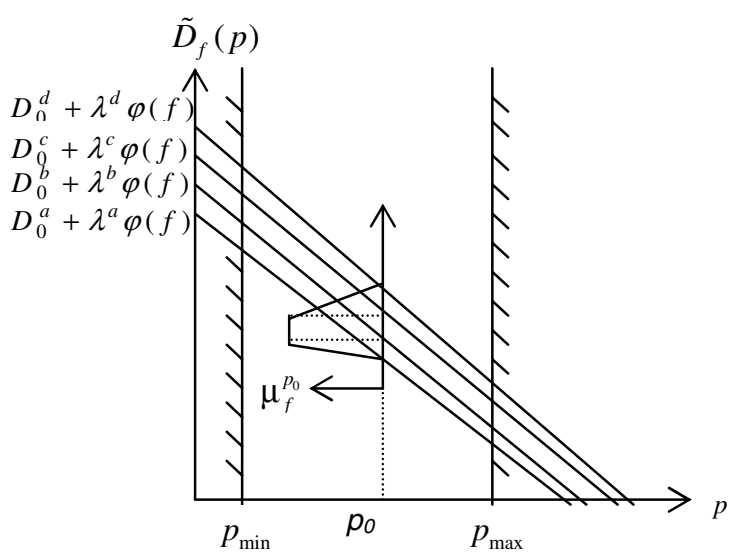

Figure 3. Fuzzy linear demand function for a given frequency $f$

\subsection{Fuzzy expression of profit}

For the operator, the fuzziness of demand propagates to the estimate of his profit resulting from a choice of the fare and the frequency. Let the fuzzy estimate of his own profit be written as $\tilde{\pi}$.

Let $\tilde{s}^{p, f}=\min \left\{\tilde{D}_{f}(p), K . f\right\}$ be the fuzzy estimation of the satisfied demand corresponding to the couple $(p, f)$; the membership function of $\tilde{s}^{p, f}$ is denoted $\mu_{s}^{p, f}$, this membership function is deduced from the one of $\tilde{D}_{f}(p)$ by:

$$
\mu_{s}^{p, f}(s)=\left\{\begin{array}{cc}
\mu_{D_{f}}^{p}(s) & \text { if } s<K f, \\
\max _{d \geq K f} \mu_{D_{f}}^{p}(d) & \text { if } s=K f \\
0 & \text { if } s>K f
\end{array}\right.
$$

Then the fuzzy estimation of the profit of the carrier corresponding to the couple $(p, f)$ is given by:

$$
\tilde{\pi}_{f}(p)=p \cdot \tilde{s}^{p, f}-C(p, f)
$$

And the membership function $\mu_{\pi}^{p, f}(\pi)$ of $\tilde{\pi}_{f}(p)$ is obtained from the one of $\tilde{s}$ as follows:

$$
\mu_{\pi}^{p, f}(\pi)=\mu_{s}^{p, f}[(\pi+C(p, f)) / p]
$$

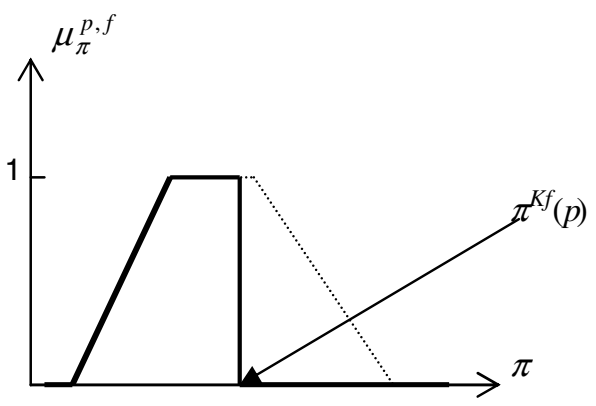

Fiqure. 4. Membership function of the profit

For a given frequency level $f$, the program of the operator who tries to maximize his profit while considering a capacity constraint is here formulated such as:

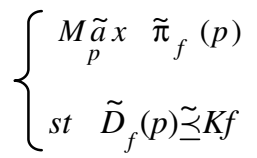




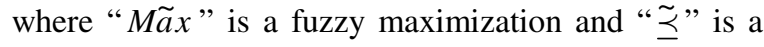
fuzzy operator associated to the above fuzzy inequality denoted $\mathfrak{I}$ with a membership function given by:

$$
\left\{\begin{array}{l}
\text { if } K . f \leq D_{f}^{a}(p) \text { then } \mu(\mathfrak{I})=0 \\
\text { if } D_{f}^{a}(p)<K . f<D_{f}^{d}(p) \text { then } \\
\quad \mu(\mathfrak{I})=\left(D_{f}^{d}(p)-K f\right) /\left(D_{f}^{d}(p)-D_{f}^{a}(p)\right) \\
\text { if } K . f \geq D_{f}^{d}(p) \text { then } \mu(\mathfrak{I})=1
\end{array}\right.
$$

Fig. 5 provides a graphical representation of the fuzzy constraint of problem (17).

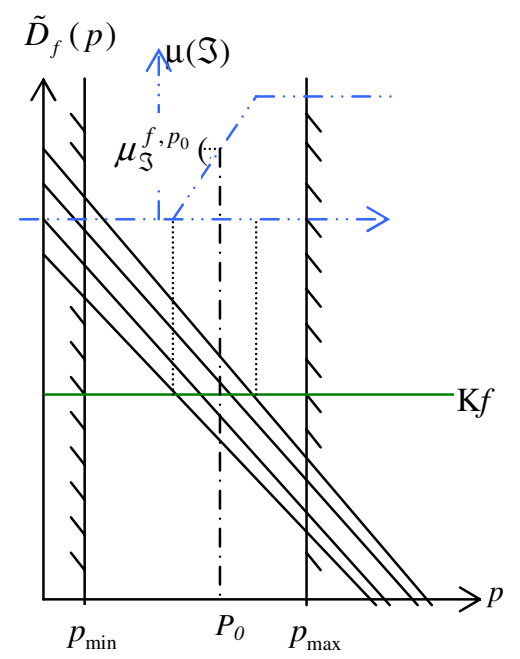

Figure. 5. Fuzzy linear demand function for a given frequency $f$ and the available capacity level

When the estimated demand is given by a fuzzy linear function, the problem (17) is a fuzzy linear programming. Several methods are given in the literature to resolve fuzzy linear programming problems ([6] and [7]) depending on the particularities of the problem (the most treated case is when the objective function is crisp and only the constraints bounds are fuzzy). In this paper, all the coefficients are fuzzy but the bounds of the constraints. That is why a method of resolution is given in this particular configuration, based on the techniques of ranking fuzzy numbers which will be treated in next subsection.

\subsection{Decision making}

The final choice of the decision maker, even based on fuzzy representations, to be practical will be a crisp one. For that, he can perform an analysis of risk (spoilage and spill ones) using the above fuzzy representation of demand. For a given frequency level $f$ and for each feasible fare $p$, a fuzzy set representative of the distribution of the estimate of the resulting profit is associated. To choose a price $p_{f}^{*}$, it should be necessary to compare the fuzzy numbers associated to the profit. Since demand is here considered to be represented by convex fuzzy sets, it will be also the case for the profit. Different techniques are available to rank convex fuzzy sets for example [5]. The main adopted approaches perform a defuzzification of the problem through a relationship that associates to each fuzzy number a crisp value. Here the defuzzyfication is making use of the barycenter of the fuzzy profit $\tilde{\pi}_{f}(p):$

if $\mu(\mathfrak{I}) \neq 0$ then

$\pi_{f}^{e}(p)=\int_{\pi_{f}^{a}(p)}^{\min \left(\pi^{K f}, \pi_{f}^{d}(p)\right.} \pi \cdot \mu_{\pi}^{p, f}(\pi) d \pi / \int_{\pi_{f}^{a}(p)}^{\min \left(\pi^{K f}, \pi_{f}^{d}(p)\right.} \mu_{\pi}^{p, f}(\pi) d \pi$

else $\quad \pi_{f}^{e}=0$

The problem is transformed into the maximization of the above non linear (and non concave) expression with respect to $p$. An optimal $\left(\pi_{f}^{e}\right)^{*}=\max _{p} \pi_{f}^{e}$ can be obtained by inspection over a discretization of the feasible set of $p$. Let $p_{f}^{*}$ be the corresponding fare.

The final decision step is to choose the frequency level denoted $f^{*}$. It is given by the expression:

$$
f^{*}=\underset{f \in F}{\arg \max }\left\{\left(\pi_{f}^{e}\right)^{*}, f \in F\right\}
$$

to which is associated the selected fare $p^{*}=p_{f^{*}}^{*}$. The expected profit is then represented by the fuzzy set: $\tilde{\pi}_{f *}\left(p^{*}\right)$.

\subsection{Risk analysis}

The main risks to which the decider is confronted are:

$\checkmark$ A risk of spoilage: when the offered capacity exceeds the effective demand level $d_{e f f}$, then aircraft will fly with empty seats (see fig.6.a).

$\checkmark$ A risk of spoilage: when the effective demand $d_{\text {eff }}$ is larger than the offered capacity, so a large number pd potential passengers will be refused at booking (see fig.6.b). 


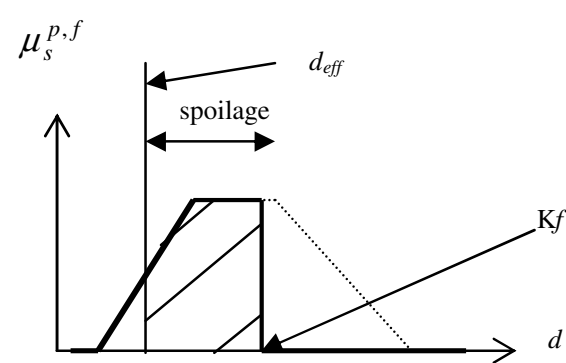

Figure.6a. Representation of risk example : spoilage

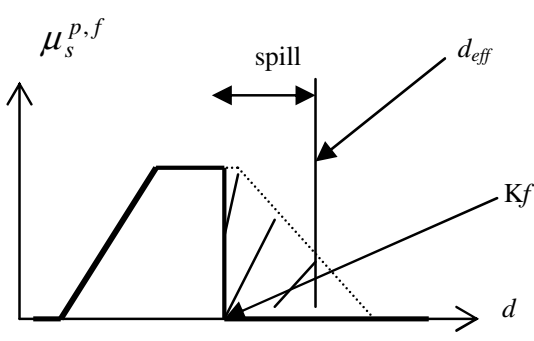

Figure.6b. Representation of risk example: Spill

The decision maker could have a preference with respect to these risks. For that, he can introduce in the defuzzyfication method given by (19) a weight expressing his preferences as below:

$$
\begin{aligned}
& \pi_{f}^{e}(p)=\mu(\mathfrak{I})^{q} \int_{\pi_{f}^{a}(p)}^{\min \left(\pi^{K f}, \pi_{f}^{d}(p)\right.} \pi \cdot \mu_{\pi}^{p, f}(\pi) d \pi / \int_{\pi_{f}^{a}(p)}^{\min \left(\pi^{K f}, \pi_{f}^{d}(p)\right.} \mu_{\pi}^{p, f} \\
& \quad \text { if } \mu(\mathfrak{I}) \neq 0 \\
& \quad 0
\end{aligned}
$$

else

Where $\mathrm{q}$ is a real number that can be chosen by the decider as follows:

- $\mathrm{q}>0$ if the goal is to avoid spoilage and empty seats.

- $\mathrm{q}<0$ if the priority is to avoid refusing demand and to satisfy consumers requests.

- $\mathrm{q}=0$ corresponds to (19) and represents an equal treatment for two risks.

Both studied approaches provide the decider with a couple of crisp values for action. The first value represents the fare of travel and the second represents the frequency of flights to be delivered to the market in order to get a somehow maximized profit. In the case of a deterministic approach, the expected profit is a crisp value while in the second approach, although the final decision is crisp, the expected profit is given by a fuzzy set. This fuzzy set displays to the decision maker a view of uncertainty in which lies the solution. Applying the proposed fuzzy approach, the experts can intervene at different stages and in several ways in the decision process. On one hand and depending on their opinion about the trend of the activity, they can decide about the level functions representing the fuzzy demand. On the other hand, they can be risk averse and choose the criteria concerning the minimization of risk in the profit maximization stage.

\section{Conclusion}

This communication has considered the case in which demand functions for airlines are given in a fuzzy way. This representation of demand seems opportune since air transportation demand is very sensitive to many unpredictable factors and airlines operate in a very imperfect information environment. It has been shown in this communication that decision making with fuzzy information can be formalized, without too much intricacy, for the monopoly situation.

Future works should be turned towards the case of competition.

\section{References}

[1] Carlsson F. Price frequency Choice under Monopoly and Competition in Aviation Markets.

URL:

http://www.handels.gu.se/epc/data/html/html/PDF/gunwpe0071. pdf , 2002 (visited on 10.10.2003)

[2] Castelli L., Pesenti R and Ukovich W. An airline based multi-level analysis of airfare elasticity for passenger demand. $d \pi$ Proceeding of the $7^{\text {th }}$ ATRS conference, July 2003-Toulouse France.

[3] Charfeddine, S., Mora-Camino F. and De Coligny M. (2004) Fuzzy linear regression: Application to the estimation of air transport demand. International Conference on Fuzzy Sets and Soft Computing in Economics and Finance (FSSCEF 2004).June 17-20, 2004, Saint-Petersburg, Russia

[4] Charfeddine S., De Coligny M., Mora-Camino F. and Cosenza C.A.N. A Fuzzy Approach of Competition on Air Transport Market. Proceeding of AMERC, july 2003, Montreal. [5] Fortemps P. and Roubens M. "Ranking and Defuzzification Methods based on Area Compensation". Fuzzy Sets and Systems, 82, (1996) pp 319-330.

[6] Rommelfanger H., "Fuzzy linear programming and applications", European Journal of Operational Research 92 (1992) 512-527.

[7] Shaocheng T. ,Interval Number and fuzzy linear programming. Fuzzy Sets and Systems, 66 (1994) 301-306.

[8] Schipper Y., Environmental Costs and Liberization in European Air Transport. A Welfare Economic Analysis. Transport Economics Management and Policy Series. General Editor : Kenneth Button. PP 198, 2001.

[9] Tanaka, H., Uejima, S. and Asai. "Linear regression analysis with fuzzy model", IEEE Transactions Systems, Man and Cybernetics 12 (1982) 903-907.

[10] Zimmermann H., Fuzzy set theory - and its applications, ch.7,8. Hardcover. PP.544, 1991. 